EPJ Web of Conferences 33, 05011 (2012)

DOI: $10.1051 /$ epjconf/20123305011

(C) Owned by the authors, published by EDP Sciences, 2012

\title{
Analytical method for estimating energy output of small wind turbines integrated in urban areas
}

\author{
M. Popovac
}

Austrian Institute of Technology, Energy Department, Gieffinggasse 2, 1210 Vienna, Austria

\begin{abstract}
This paper presents the development of the analytical approach for estimating the wind energy potential at locations around buildings in urban areas that are suitable for installing Small Wind Turbines (SWT). This development is performed in three steps. In order to analyze the flow pattern in a typical urban geometry, the first step consisted of a series of numerical simulations, where an assumed urban configuration (specified building size and respective distances between the buildings) was varied with an incremental value, and for each analyzed configuration different incoming wind conditions (different reference wind velocity) were incrementally imposed. In the second step the velocity profiles in the characteristic cutlines around the central building under investigation were extracted from the respective realizations of urban flow numerical solutions, and an analytical expression was derived approximating all extracted velocity profiles to fit the best an assumed flow pattern. The derived analytical expression was cross-plotted with the results of a fully three-dimensional realistic urban flow numerical solution, and the obtained matching level was satisfactory. Finally, using the derived expression, in the third step the estimation of SWT energy output was defined based on the average wind velocity information at given location and the related urban configuration characteristics.
\end{abstract}

\section{Introduction}

In an attempt to improve the overall energy efficiency in buildings, the idea of integrating Small Wind Turbines (SWT) in building envelopes is gaining popularity despite many open issues related to it. One of the doubts regarding SWT integration in urban environments is a limited energy potential of the wind in urban areas, and in order to promote the SWT integration in urban areas it is necessary to develop a simple tool which can be used for a quick estimation of wind energy potential. The problem with developing such a tool is that the urban flow pattern is characterized by a number of mutually interacting flow features (e.g. separation, reattachment, impingement etc.) in connection with very complex geometry. This is why the exact prediction of the urban flow velocities is a very demanding task, which is typically accomplished by means of Computational Fluid Dynamics (CFD) simulations. However, these simulations can be very lengthy, and thus they are not appropriate for quick estimations. In order to develop a tool which can be used for a quick estimation of the wind energy potential in urban environments, a generalization based on the assumptions of the urban flow pattern needs to be performed using the results of the CFD simulations. Such a tool will be very helpful not only for the economic analysis of installing SWT in urban areas, but also for analyzing e.g. the energy supply measures in the early stages of the urban planning. 
Through the generalization based on the assumed pattern of the urban flow field, this tool is taking into account the averaged wind conditions at given location, as well as the main parameters of the urban morphology. These include the size of buildings and their arrangement, defining thus the aerodynamic properties of the urban flow pattern. Due to its simplicity and applicability, this analytic method fits perfectly into a tool for the urban planning in regards to SWT in urban environments. Nevertheless, the user needs to pay attention to the accuracy of the derived analytical estimation method, because of the assumptions introduced for developing this analytical method. The sections that follow present the derivation of this analytical method and its application, concluding this paper with a short discussion and future prospects.

\section{Wind velocity analytical expression}

The development of the analytical approach for the prediction of the wind energy potential was performed in three steps [1]. In the first step a series of numerical simulations is performed for a typical urban geometry: an assumed configuration (specified building size, as well as the distance between the buildings) was varied with specified incremental value, and for each analyzed configuration different incoming wind conditions (different reference wind velocity, and different wind direction) were subsequently imposed. Shown in Fig. 1 is only a small part of these realizations, because the total number of combinations counts the variations in building size, the building arrangement, and the wind conditions. For each of these combinations the converged solution of the numerical simulation was obtained in order to analyze the established flow pattern.
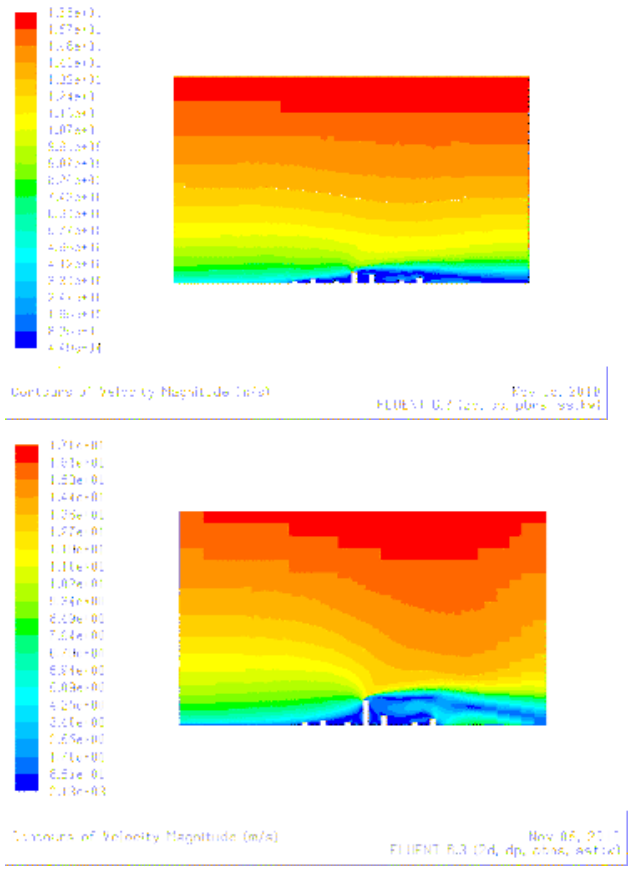
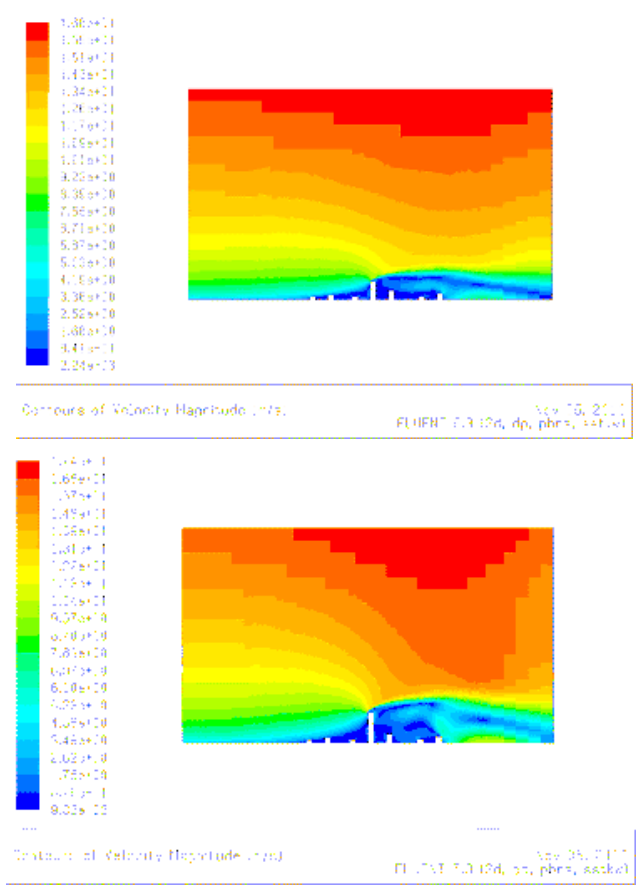

Fig. 1. The urban geometry variation (selection of the realizations) and the corresponding urban flow pattern.

The main aim here is to derive an analytic expression for the wind distribution above the building where SWT is to be installed, and to that purpose the velocity profiles in the characteristic cutlines around the central building under investigation have been extracted from the urban flow solutions for respective numerical simulation realizations. The basic idea is to devise an analytical expression 


\section{$2^{\text {nd }}$ European Energy Conference}

which will approximate all extracted velocity profiles the best, and this task has been performed in the second development step. For this derivation the type of analytical expression that is sought has to be assumed, i.e. the assumption about the established flow pattern has to be made. In the present work, as shown in Fig. 2, the representation of the urban flow pattern which is the most widely used in literature has been adopted. This includes the logarithmic incoming wind profile (with $u_{p}$ as the reference velocity related to the field roughness $z_{O P}$ ), and the boundary layer in the urban environment divided in to the internal and external part (as defined with the boundary layer thickness $h_{i}$ ). Furthermore, for the characterization of the flow within the urban area, the displacement thickness $d$, and the urban roughness $z_{0 R}$ are defined (semi)empirically as functions of the geometric quantities of urban area: the most important ones are the height of the central building under investigation $h$ and the percentage of urban area occupied by buildings $A_{h}$. The values and/or expressions for these quantities have been taken from the literature [2], and summarized in Table 1. In order to come to the unique set of values for given parameter, based on the great set of extracted velocity profiles, the least square method was used.

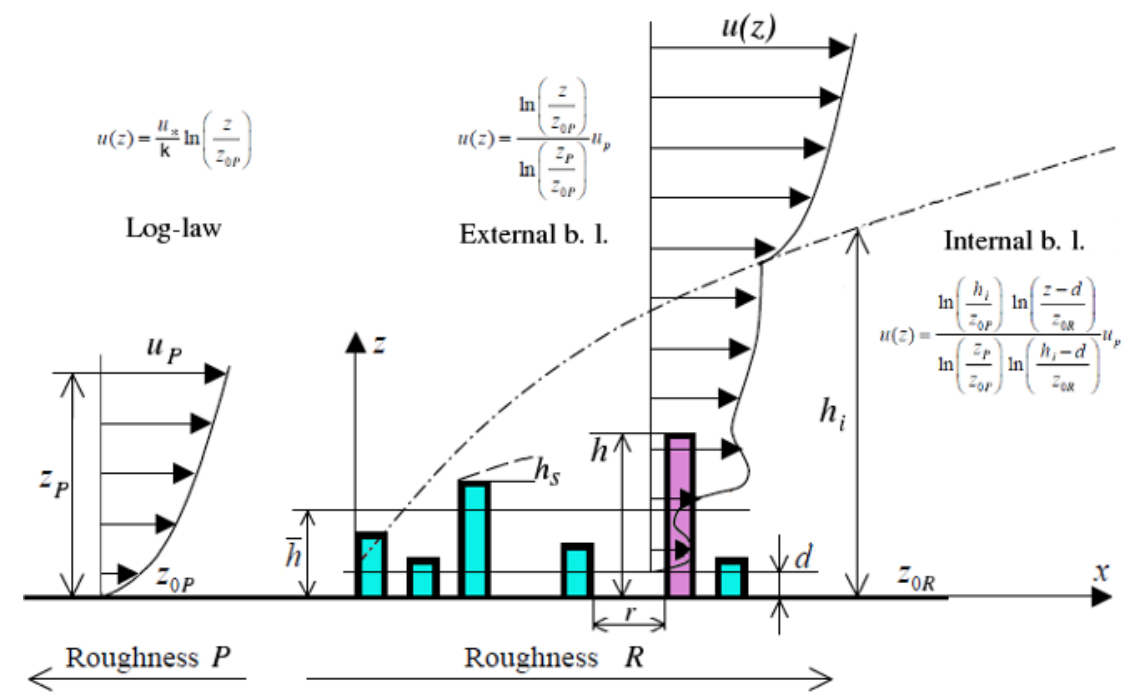

Fig. 2. The sketch of the assumed urban flow pattern, and the characteristic urban geometry parameters.

In order to downscale the assumed flow pattern to the heights comparable to the height of the building where SWT is to be installed, the correction factor $\psi$ is introduced as a function of the geometrical quantities related to the central building under investigation. The expression for $\psi$ is given in Table 1, and the final expression for the effective velocity above the roof building reads [3]:

$$
U_{\text {eff }}(z)=\frac{u_{p}}{\psi} \frac{\ln \left(\frac{h_{i}}{z_{0 P}}\right) \ln \left(\frac{z-d}{z_{0 R}}\right)}{\ln \left(\frac{z_{P}}{z_{0 P}}\right) \ln \left(\frac{h_{i}-d}{z_{0 R}}\right)}
$$

with the introduced correction factor accounting for the flow effects of the immediate surrounding on the flow pattern around the central building under investigation.

After the deriving procedure was completed, it was very important to test the derived expression. To that purpose, Eq.(1) has been cross-plotted with the results of the realistic urban flow solution [4], and Fig. 3 shows the obtained matching. The green and blue lines (full and dotted lines with symbols) indicate the range for the velocity profiles obtained from CFD urban flow simulation for different wind directions, while the read lines (full and dot-line-doted lines without symbols) represent Eq.(1) with and without the correction factor $(\psi=1)$. It can be seen that the matching of the profiles is 
satisfactory for the zone above the roof at which it is expected to install SWT (approx. $8 m$, indicated in Fig. 3 with the symbols: circle and square respectively). Of course, for the lower parts of the profile the matching is reduced, however this is still acceptable since the present work has been specifically concentrated on the zone where SWT is to be installed (the least square minimization of error has been selected exactly for the point where it is assumed that SWT will be installed).
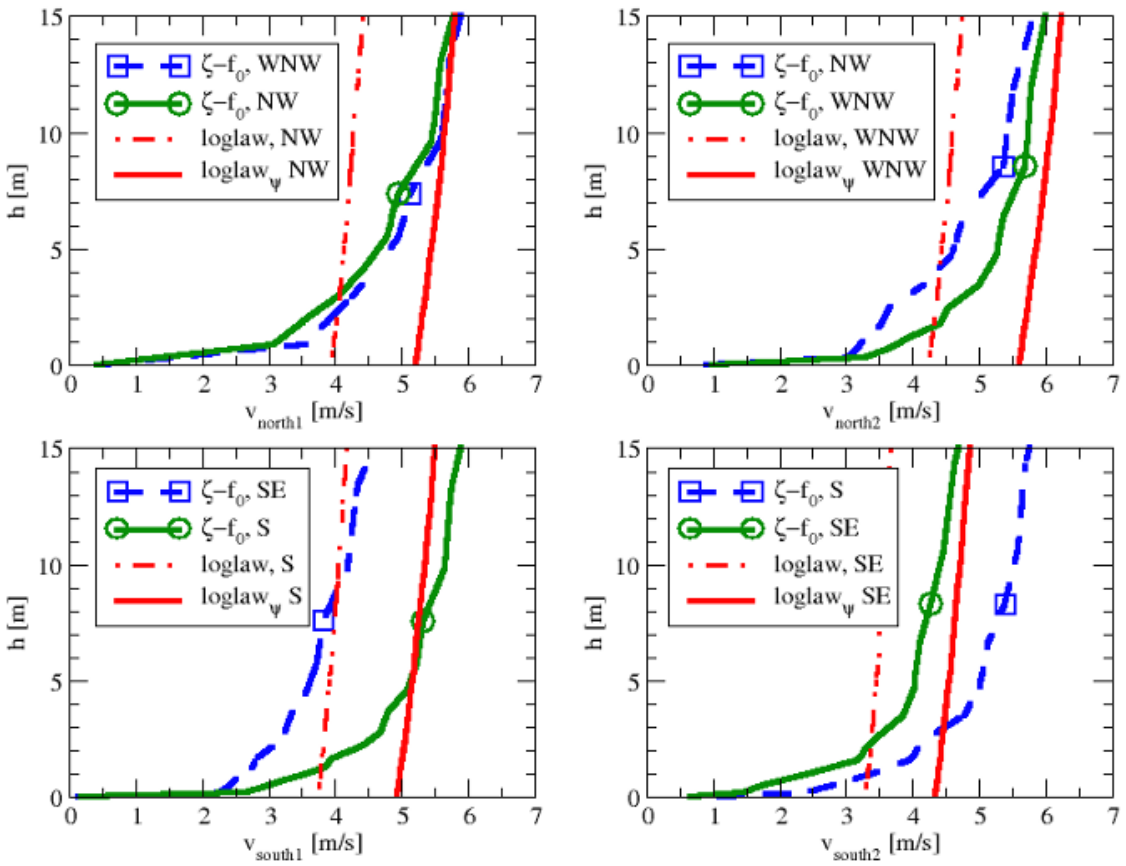

Fig. 3. The comparison between analytical expression and velocities obtained from CFD urban flow simulation.

Here it needs to be noted that with the analytical approach the accuracy is always an issue, and therefore one needs to take the results with care. Namely, although the solution was sought by analyzing very large number of urban flow realizations (varying both the geometry and the wind conditions), the assumptions made about the established flow field pattern inherently limit the generality of such an approach.

\section{Wind energy output}

Starting from the analytical expression for the effective wind velocity profile in urban areas, given by Eq.(1), in the last step of developing the method for estimating the wind energy potential one needs a statistical model for the mean wind velocity at given location. Widely used model for the variations in wind speed is the two-parameter Weibull distribution, which describes the variation of the average velocity magnitude $U$ through the scale parameter $c$ (related to the mean velocity) and the shape parameter $k$ (related to the location where the wind is analyzed). Adopting this model, the $3^{\text {rd }}$ raw moment of the Weibull distribution defines the effective wind energy flux:

$$
E_{\text {eff }}=\frac{1}{2} \rho\left\{c^{3} \Gamma\left(1+\frac{3}{k}\right)\right\}=\frac{1}{2} \rho\left\{\left[U_{e f f} / \Gamma\left(1+\frac{1}{k}\right)\right]^{3} \Gamma\left(1+\frac{3}{k}\right)\right\}
$$

where $\rho$ is the air density, and $\Gamma$ is the Gamma function. 
$2^{\text {nd }}$ European Energy Conference

Table 1. The calculation sheet for the analytical prediction of the SWT energy output.

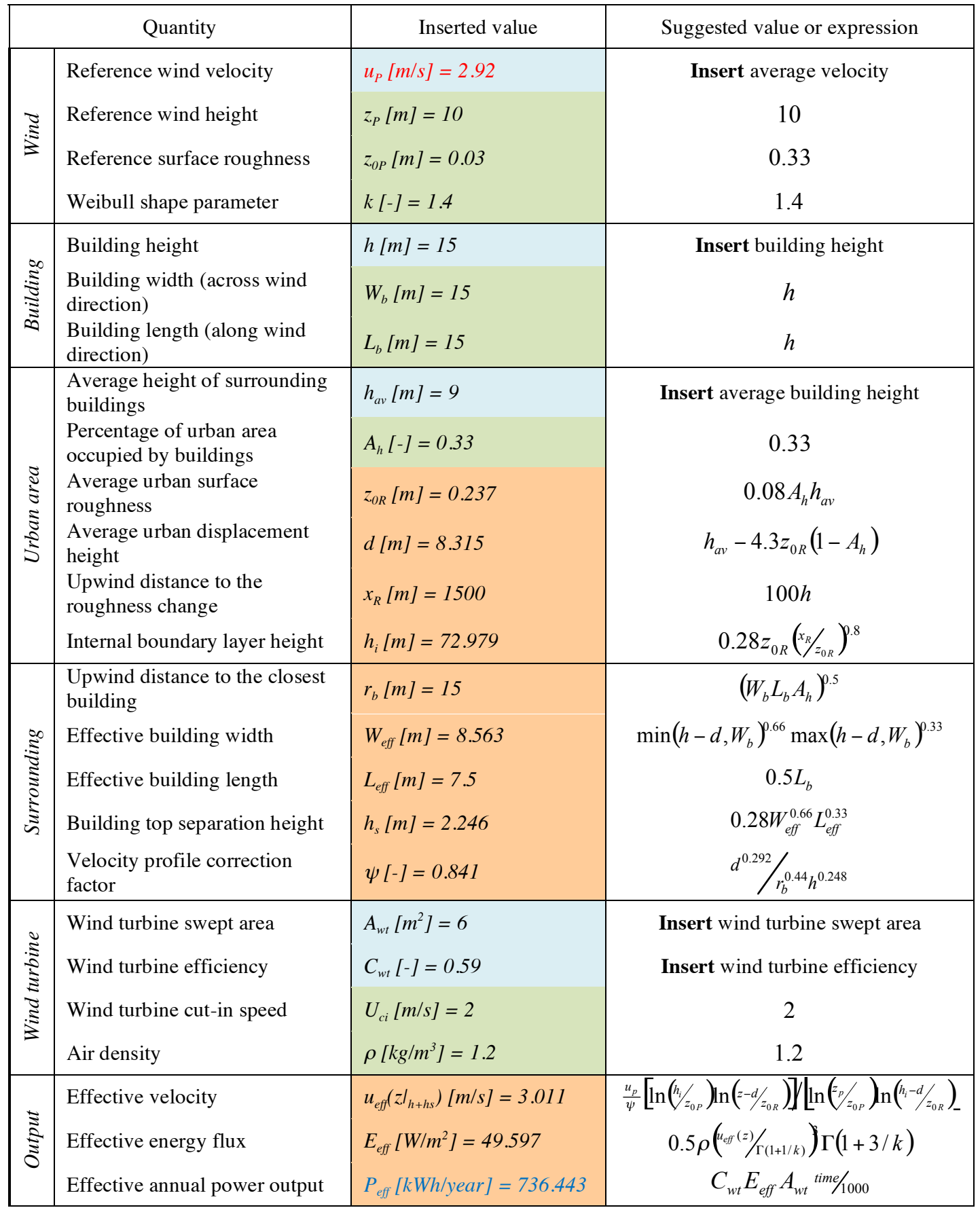

Having all the expressions defined previously, the estimation of the wind energy potential reduces to a few simple sub-steps, as summarized in Table 1 (the symbols referring to Fig. 2). By filling in the required data logically (as indicated in the first column of Table 1: first about the wind, then about the building, neighborhood and immediate surroundings, and finally about SWT itself), the user is guided to the prediction of the SWT energy output at the specific location. Some of the needed data are expected to be known, and these are highlighted with blue background (also noted with the word 
Insert, e.g. the wind velocity $u_{p}$, or the central building height $h$ ). The rest of the necessary input data can be either estimated (highlighted with the green background, e.g. the average building height $h_{a v}$, or the percentage of urban area occupied by buildings $A_{h}$ ) or calculated (highlighted with the yellow background, e.g. the urban roughness $z_{0 R}$, or the displacement height $d$ ). Their suggested values and (semi)empirical expressions, given in the last column of Table 1, can be found in the literature [2]. The most influential data are those related to the wind, since Eq.(2) shows that the wind velocity comes to the power three in the expression for the wind energy output. Therefore it is very important to reach the acceptable accuracy level for wind velocity, hence it is expected these to be either measured on site or taken from some reliable sources (e.g. wind atlas).

\section{Conclusions}

Due to recent developments in energy and environmental policy worldwide, there is a constant growth of interest for the wind energy as the supply source, together with positive market developments for the renewable energy sources in general. This paper presented an analytical method developed for the estimation of the wind energy potential in urban areas. This method can be directly used for the economic analysis of the building integration of SWT (for which the wind energy output is one of the main input parameters), hence the groups for which it can be interesting are ranging from the individual consumers interested in installing SWT on the roofs of their houses, to the urban planners who are analyzing different scenarios on the city scale for introducing renewable energy sources into the energy supply systems. Finally, the developed method can be incorporated into some other tools which are more complex (e.g. for the urban planning). This step, however, belongs to the future work, together with the accuracy improvement of the wind energy predictions.

Namely, in the core of this approach is the simplicity and applicability of the analytical method for the wind energy prediction in urban areas. Having developed such a simple method, it is not difficult to integrate it into a tool which can automatically feed in required data: the final outcome here is a simple table which can easily be turned into an Excel sheet or integrated into a Geographic Information System (GIS) module, and subsequently developed further into a tool for assessing the economic potential of installing SWT in urban environments. By incorporating the analysis of the wind energy potential in urban environments, the extension is being made towards including more renewable energy sources in urban planning.

Clearly, the accuracy issue of can be addressed by introducing a more sophisticated assumption for the established urban flow pattern. This implies the introduction of additional parameters that characterize urban morphology, as well as the non-equilibrium wind flow conditions. The example of this could be different building shapes (e.g. roofs with different inclination angles), which was in the present work simplified as the height above the roof at which a small wind turbine is to be installed. However, this also implies (as described earlier) much more time consuming 3D simulations, much more data processing, and much more validation work (hence, these issues belong to the future improvements of this basic method).

\section{References}

1. M. Popovac, D. Reiterer, E. Sarugg, STEP-A Project Report. Österreichische Forschungsförderungsgesellschaft $m b H(F F G)$, project no. 825371 (2012).

2. S. Mertens, Wind energy in the built environment - concentrator effects of buildings, Ph.D. Thesis TU Delft, (2006).

3. M. Popovac, Modeling and simulation of turbulence and heat transfer in wall-bounded flows, Ph.D. Thesis TU Delft, (2006). 
$2^{\text {nd }}$ European Energy Conference

4. M. Popovac, D. Reiterer, M. Broser, IPPONG Project Report. Österreichische Forschungsförderungsgesellschaft $m b H(F F G)$, project no. 821910 (2011). 